\title{
Comparison of two methods for subchannel seizure in GSM cell using VAMOS technics
}

\author{
Aleksandar Lebl, Dragan Mitić, Žarko Markov, Mihailo Stanić*
}

\begin{abstract}
This paper considers two methods for voice channel seizure in one GSM cell, which uses VAMOS technics for traffic efficiency improvement. An important characteristic for models comparison is call loss caused by unsuccessful connection pairing in one timeslot. The first method gives priority to voice connection quality, and the other one to traffic efficiency. The methods are estimated by the simulation, which is first tested by comparing its results to the calculation results for a very small system. The influences of traffic intensity, allowed power difference of two paired connections and attenuation coefficient on the loss caused by unsuccessful pairing are assessed.

K e y w or d s: GSM, VAMOS, pairing, connection loss
\end{abstract}

\section{Introduction}

VAMOS technics (voice service over adaptive multiuser channels on one slot) significantly contributes to the traffic capacity of classical GSM cell.It is a relatively new technics, presented in the very actual reference devoted to mobile systems, [1]. This technic is implemented in GSM cell with frequency (FDM) and time (TDM) multiplex, but each time channel may be used for two full rate, FR, connections (over two OSC, orthogonal subchannels). In the case of full rate connection transmission capacity is doubled, and in the case of half rate connection transmission it is quadrupled. This capacity increase is based on QPSK (quadrature phase-shift keying) modulation and orthogonal training sequence codes (TSC) implementation, [2], [3]. This modulation (ie its variant AQPSK or $\alpha$ QPSK) is implemented in downlink (direction) operation, when signal is sent to both mobile station (MS) users in the same time over the same channel. $\alpha$ QPSK modulation implementation from base station (BS) to MS allows the signal to be sent by different power to different MS, who use the same channel wit a different attenuation. In this way each MS receives the signal of approximately same power. The process of two connections placing in one timeslot is called pairing. The main limitation in the pairing process is that the subchannel power imbalance ratio (SCPIR) of the two connections using the same timeslot, is smaller than $\Delta_{\max }(\mathrm{dB})$, [4]. Two methods of OSC seizure are compared: one, which gives priority to connection quality and the other, which gives priority to better traffic utilization. We present a model of GSM cell and the pairing technic dealing with the first and second pairing procedure in the same timeslot. These are compared and numerical examples are presented. The short description of the traffic process simulation, which is used for estimation of models efficiency, is given eventually.

\section{Model, assumptions and designations}

Let us consider a circular GSM cell of radius $R$, where VAMOS technics is implemented. User distribution in the cell is uniform, ie the number of MSs per cell unit area is constant. All MSs have possibility to use VAMOS technics. The control of emission power is used in base station (BS) in the direction towards user mobile stations. Distance $(r)$ between $\mathrm{MS}$ and $\mathrm{BS}$, is $0 \leq r \leq R$, and signal attenuation, which depends on distance MS$\mathrm{BS}$, is $a=k r^{\gamma}$, where $k$ is coefficient of proportionality and $\gamma \leq 25$, [5]. The number of traffic timeslots (TCH) is $N$ and the number of MSs is $M$. The channels are used for telephone traffic (with full rate, FR) which means that after a call arises in a random moment, the connection must be established in relatively short time interval, [6]. Each user, ie MS initiates new call with the same intensity. That is why the total offered traffic $A=\lambda / \mu$ is constant. The connection duration is random variable with negative exponential distribution and the mean value $t_{m}=1 / \mu$, while $\lambda$ is total call intensity. It is supposed that $N<<M$. The considered model is pure VAMOS, [7], where all active MSs use VAMOS technics, ie the maximal number of connections is $2 N$.

Traffic serving is with loss, meaning that this is full availability traffic model. Serving model in this case, when VAMOS technics is used, is not Erlang model because, due to pairing, the assumption that each call can seize each idle (sub)channel is not satisfied. In this paper the states of classical Erlang model are designated by $(j, A, 2 N), j=0,1, \ldots, 2 N-1,2 N$ The probability that all channels are busy in Erlang model is denoted by $B_{E}=P(2 N, A, 2 N)$. The state probabilities in the considered VAMOS model are denoted by $P j, j=0,1, \ldots, 2 N-1,2 N$, and the state probability that all channels are busy is $B=P\{2 N\}$. The signal

Institute IRITEL a.d., Batajnički put 23, 11080 Belgrade, Serbia, lebl,mita,zmarkov,mihailo@iritel.com 


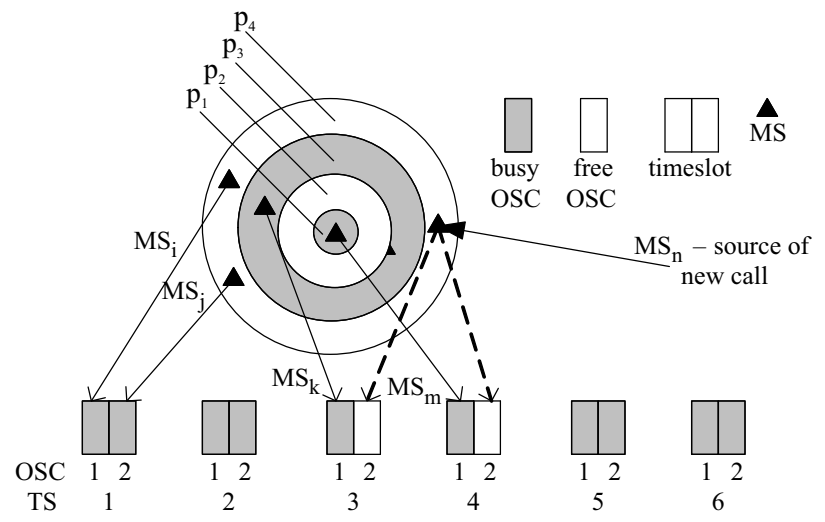

Fig. 1. Successful and unsuccessful pairing

(a)
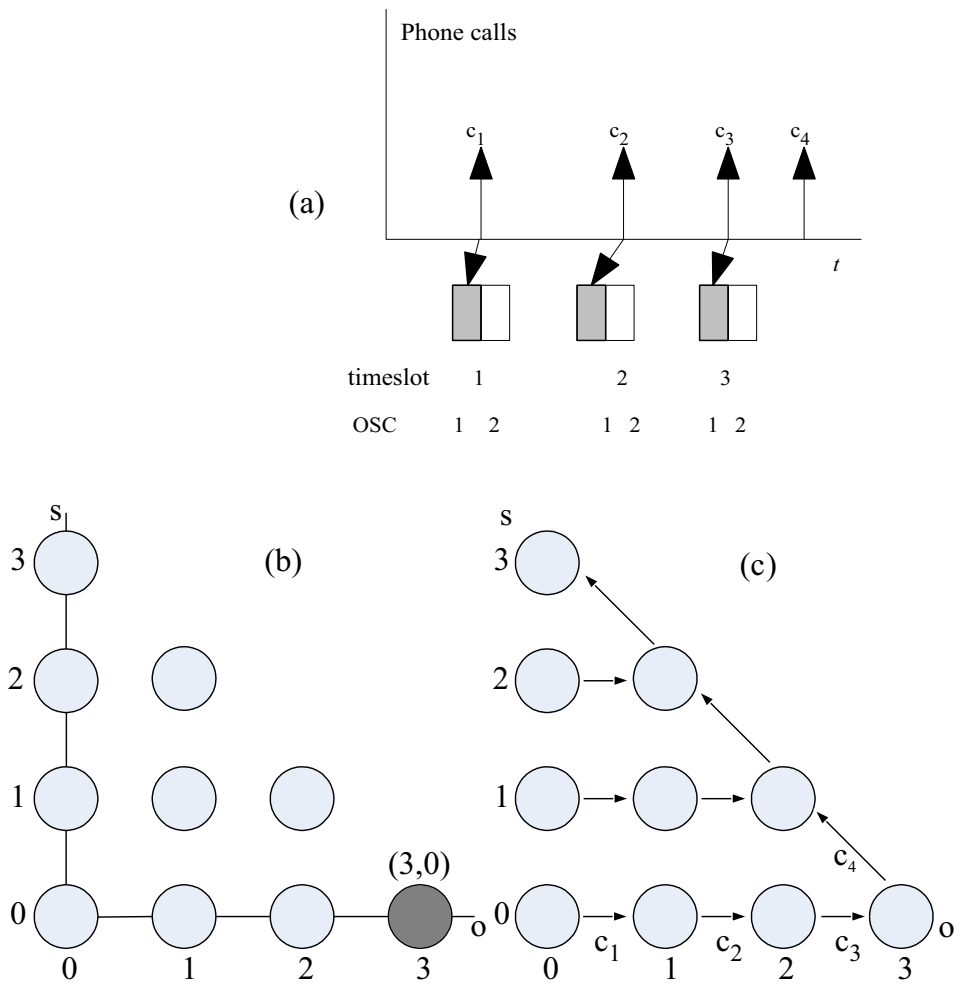

(d)

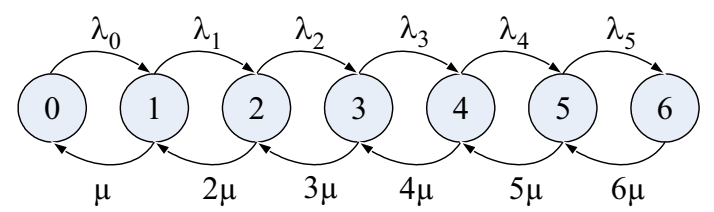

Fig. 2. The first method: (a) - idle OSC seizure, (b) - possible states, (c) - birth process, and (d) - transition diagram

power difference, which is assigned to the users with different attenuation, is denoted by $\Delta$. The power, which is sent from BS towards MS, is $P_{\min } \leq P \leq P_{\max }$. The BS power control is performed in 15 steps of $2 \mathrm{~dB},[8],[9]$.

\section{Pairing}

Pairing is realized in such a way that in one channel are grouped two connections, for which necessary power does not differ for more than $\Delta l$, users $\mathrm{MS}_{i}$ and $\mathrm{MS}_{j}$, Fig. 1. In this figure the group of 6 timeslots were considered, and 4 of them are busy by paired connections. Two timeslots (TS3 and TS4) are half busy, ie only one OSC is busy. In random moment new call is initiated from the user $M S_{n}$. The necessary power for $M S_{n}$ is greater than the necessary power for OSC1 in TS3 and OSC1 in TS4 for more than $\Delta l$ ie $\left|P_{n}-P_{k}\right|>\Delta l$ and $\left|P_{n}-P_{m}\right|>\Delta l$. The new connection pairing in timeslots TS3 and TS4 
(a)
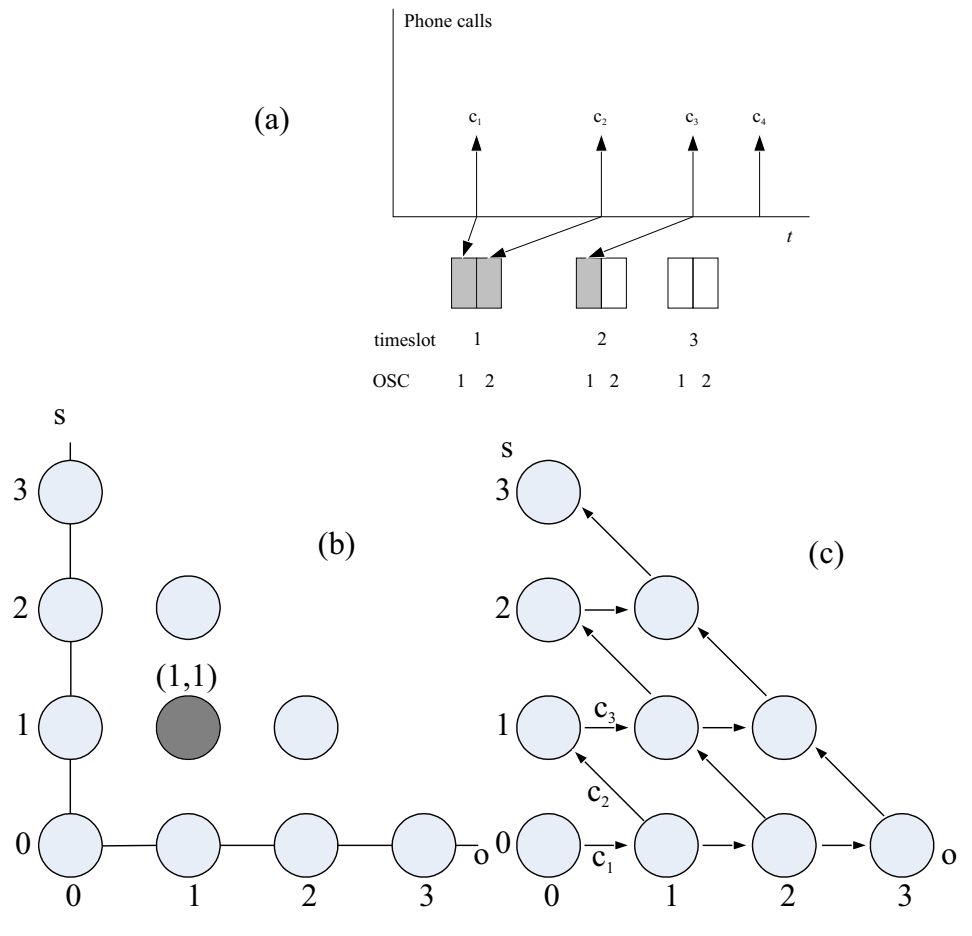

(d)

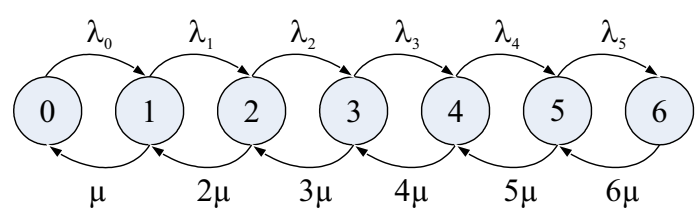

Fig. 3. The second method: (a) - idle OSC seizure, (b) - possible states, (c) - birth process, and (d) - transition diagram

is unsuccessful in this case. It is considered in this paper that the new call, which canot be paired with any existing one, is lost due to unsuccessful pairing (unpairing). The probability of unsuccessful pairing will be denoted as $B_{p}$.

\section{The first method of OSC assignment}

It is known that inter-channel interference (ie mutual influence of OSCs situated in one timeslot) canot be avoided when two voice channels are placed in the same timeslot. As the consequence the quality of voice signal is degraded. Thats why the main rule for the first model of OSC assignment to the new call is: the new call is placed in one subchannel of idle timeslot if it exists. Two voice connections pairing in one timeslot begins when there are no completely idle timeslots. Fig.2, for simplicity, presents hypothetical group of only three timeslots. Channel assignment to new calls c1, c2 and c3 according to the first model is presented symbolically in Fig. 2(a).

Let us suppose that all timeslots (3 of them), ie OSCs (6 of them) are idle. New calls c1, c2 and c3 seized one (the first) OSC in each timeslot. The fourth call (c4) must pass pairing procedure, ie confirmation that its necessary power and a power of existing call in a timeslot is not greater than $\Delta_{\max }=\Delta l$. If successful pairing canot be achieved in any of three half-busy timeslots, the call is considered to be lost due to unpairing.

Let us denote by $o$ the number of busy OSCs where the second OSC in the same timeslot is idle and let $s$ be the number of timeslots where both OSCs are busy. The state determined by these two numbers is denoted as $(o, s)$. Then $p(o, s)$ is probability that $o$ half-timeslots and $s$ full-timeslots are busy. It is obvious thatq total number of busy OSCs is $o+2 s$. The state from Fig. 2 may be denoted as (3,0), Fig. 2(b), ie this state probability as $p(3,0)$. The state diagram of new call arrival (birth process) in the first model is presented in Fig. 3(c)).

Figure 2(d) presents state diagram, where the variable is the number of connections. Let us note that the call intensity $\lambda$ at $j=0,1,2$ is constant, while its effective value at $j=3,4,5$ is smaller due to unsuccessful pairing. The state with three busy OSCs will more often be $(3,0)$ than $(1,1)$ when this method is implemented.

\section{The second method of OSC assignment}

The following rule is valid for the second method of OSC assignment: idle OSC in a timeslot, which is half busy (if it exists), is dedicated to a new call. In this way idle timeslots are spared and partly busy timeslots are 


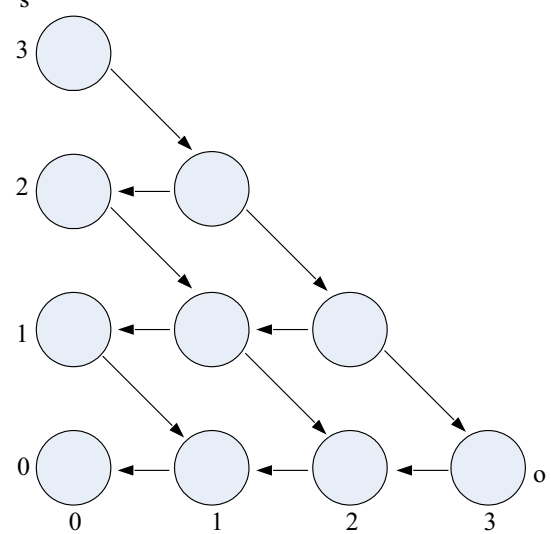

Fig. 4. Death process for both methods

completely seized. This method allows decreasing probability of unsuccessful pairing. An example of this second method is presented in Fig. 4. After three calls have arrived, a system state is $(1,1)$. It is obvious that if the call c4 is unsuccessfully paired, one idle timeslot exists for call c4 realization.

The system state from Fig. 3(a) is emphasized on the diagram of possible states in Fig. 3(b). After that, the state diagram in a case of call arrival (birth process) for the second model is presented in Fig. 3(c) and the state diagram with the number of realized connections as a system variable is presented in Fig. 3(d).

The state diagram for call ending (death process) is presented in Fig. 4 and it is valid for both processes.

The characteristic of OSC seizure in a case of the second method is that a state with three busy OSCs is more often $(1,1)$ than $(3,0)$.

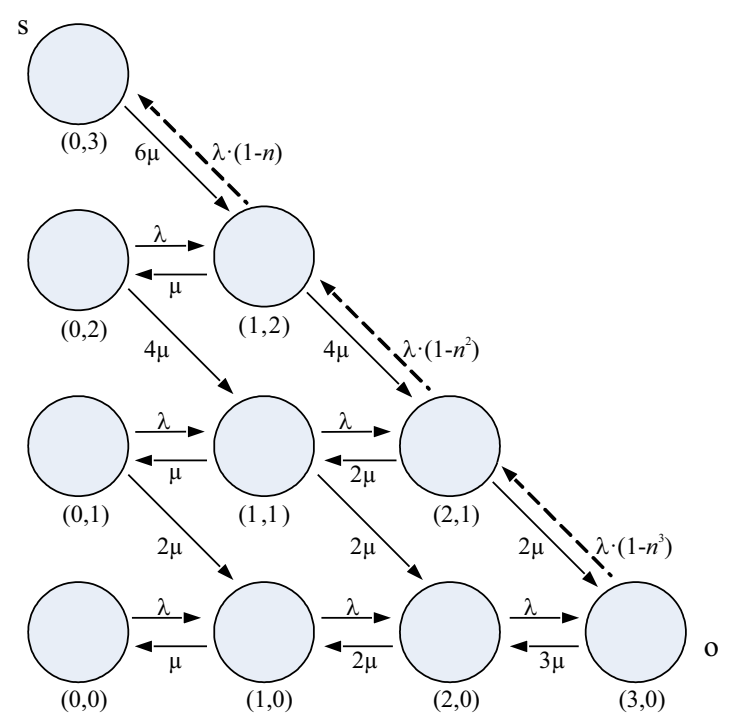

Fig. 5. Birth-death process for the first method

\section{Methods comparison}

It is clear that in the first case inter-channel interference, ie OSCs mutual influence, is significantly smaller. That is why voice signal quality is better. An advantage of the second method is greater efficiency, ie smaller probability of unsuccessful call pairing. Two-dimensional birthdeath processes may be presented if figures 2(c) and 4 are consolidated in Fig. 5 for the first method, and Fig. 3(c) and 4 in Fig. 6 for the second method.

According to Kolmogorov criterion, [9], it is obvious from figures 7 and 8 that traffic processes for both presented systems are not reversible. It means that it is impossible to simply calculate using cut equations system state probabilities, ie probabilities of successful service for the systems with greater number of timeslots. Thats why these models will be tested in two steps.

The first step is analytical and simulation description of a model with small number of timeslots. Such a model cant be found in practice, but it allows calculation of state probabilities. This step is used to prove accuracy of simulation method. The results of simulation are compared to the results of calculation in a case of small models consisted of three timeslots (6 OSCs), that are presented in Fig. 5 and Fig. 6. It is found a good agreement of system state probabilities for calculation and simulation results. In this way simulation model is verified and, based on this result, it is possible to realize the second step of an analysis: testing characteristics of two methods for idle OSCs assignment in a case of greater system implementation only by simulation model.

Dashed lines with arrows in Fig. 5 and Fig. 6 present state transitions realized by call pairing.

It is obvious from Fig. 5, which represents transition diagram for the first method, that each transition

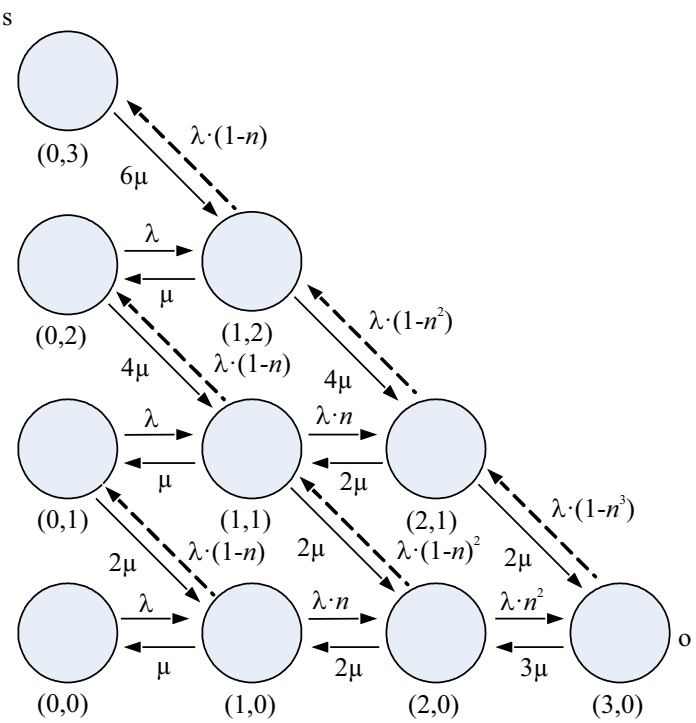

Fig. 6. Birth-death process for the second method 
that requires pairing: $(3,0) \rightarrow(2,1),(2,1) \rightarrow(1,2)$ and $(1,2) \rightarrow(0,3)$ may be finished by unsuccessful pairing.

In Fig. 6, which represents the second method, there are 6 such transitions: $(1,0) \rightarrow(0,1),(2,0) \rightarrow(1,1)$, $(1,1) \rightarrow(0,2),(3,0) \rightarrow(2,1),(2,1) \rightarrow(1,2)$ and $(1,2) \rightarrow(0,3)$. As a difference from the first method, the first three transitions in a case of unsuccessful pairing may be realized, in a case of unsuccessful pairing, by seizing one OSC in an idle timeslot. In a case of last three transitions $(3,0) \rightarrow(2,1),(2,1)) \rightarrow(1,2)$ and $(1,2) \rightarrow(0,3)$ call realization may be finished by unsuccessful pairing. According to this analysis, it is expected that the efficiency of the second method would be greater than the first one.

Intensities of new request generation are denoted by $\lambda$ in Fig. 5 and Fig. 6. As it is $N<<M$, this intensity is the same in each system state. The call ending intensity of an established connection is . In a case that connection is realized using pairing, new request generation intensity is modified (multiplied) by the probability of successful pairing. For example, in state $(i, j)$ there are $i$ possibilities for pairing. In that case, probability of successful pairing will be $\left(1-n^{i}\right)$, where $n$ is probability that pairing may not be realized on one of timeslots. Let us now consider situation for the second method, Fig. 6, when there are $N-j,(j<N)$ idle timeslots. If pairing may not be realized in such a system, a new generated request will seize OSC in one of idle timeslots. Probability of successful pairing is $(1-n i)$. Connection realization over OSC in idle timeslot is a consequence of unsuccessful pairing and this probability is $n^{i}$.

Base station emission power is adjusted in steps of 2 $\mathrm{dB},[8],[9]$, depending on distance between base station and mobile user. The whole area of base station cell is divided in 15 subareas (annuli). A base station sends signal of same power to users situated in each of 15 annuli. If a probability of successful pairing is denoted as $P$ and $P_{i}$ is probability that new generated request is in one of annuli for which pairing with the existing request from area $i, i=1,2, \ldots, 15$, may be realized, probability of unsuccessful pairing $P_{n}=1-P$ is determined by an expression

$$
P_{n}=1-\sum_{i=1}^{15} P_{i}^{\prime} P_{i}
$$

where $P_{i}^{\prime}$ is probability that the first user is in area $i$ (one of 15 annuli). The probability $n$ ??? in Fig. 7 and 8 corresponds in this case to the value determined by (1).

\section{Examples}

Let us consider two GSM cells where VAMOS technics is implemented. There are 6 timeslots, ie 12 OSCs in each cell. The first method is implemented in the first cell and the other in the second cell. The loss due to the lack of idle OSCs $(B)$ and the loss due to the unsuccessful pairing $\left(B_{p}\right)$ are considered. Variables related to the first (the second) method are denoted by index 1 or 2 .
Figure 7 show $B$ and $B_{p}$ as functions of the offered traffic (A). In this case the allowed power difference between two paired connections is $\Delta l=4 \mathrm{~dB}$ and the propagation coefficient is $\gamma=3$.

It can be concluded from Fig. 7 that the loss, caused by the lack of idle channels, is approximately equal, but the second method is more efficient when considering loss due to unpairing. The loss difference is several percent and this difference is decreased when traffic is increased.

Figure 8 presents results of simulation for the same mobile cells as in Fig. 7. Here is presented total loss $\left(B+B_{p}\right)$ for both methods in the cells, which use VAMOS technics. These results are compared to the classical Erlang loss $\left(B_{E}\right)$ in a group of 12 channels. In this example it is also $\Delta l=4 \mathrm{~dB}$ and $\gamma=3$.

Figure 9 presents influence of allowed power difference between two connections which are paired, $\Delta_{l}$, on the loss. The cell with the same characteristics as in Fig. 9 and 10 is analyzed. The allowed power intervals for pairing $\Delta_{l}=4 \mathrm{~dB}$ and $\Delta_{l}=10 \mathrm{~dB}$ are considered. When the allowed power difference $\Delta_{l}$ is increased, total loss is decreased. This fact is logical. It is possible to prove that loss due to pairing decreases, while the loss due to the lack of idle resources slowly increases. It can be shown that it is

$$
\begin{aligned}
& \lim _{\Delta_{l} \rightarrow 30 d B}\left(B_{1}+B_{p 1}\right)=\lim _{\Delta_{l} \rightarrow 30 d B}\left(B_{2}+B_{p 2}\right)= \\
& =B_{E}=\frac{\frac{A^{N}}{N !}}{\sum_{i=0}^{N} \frac{A^{i}}{i !}}
\end{aligned}
$$

because when power difference is, hypothetically, increased to $\Delta_{l}=30 \mathrm{~dB}$ all connections may be paired and the loss due to unsuccessful pairing disappears, ie the model becomes classical Erlang model.

Figure 10 presents influence of environmental attenuation coefficient $\gamma$ on loss. The increased attenuation coefficient increases loss due to unsuccessful pairing. General feature of the first method for idle channel assignment remains unchanged: the first method causes greater loss. This difference decreases when offered traffic increases.

\section{Simulation}

Simulation programs are very powerful tool for testing and proving traffic characteristics of different telecommunication systems, including mobile telephony systems. The program, developed for an analysis in this paper is based on experience from appropriate programs, presented in [11]-[14]. When considering these programs, the most general flow-chart has the program presented in [11] and the simulation developed for VAMOS systems analysis is modification of this program. The program is written in $\mathrm{C}$ programming language. One simulation trial is 


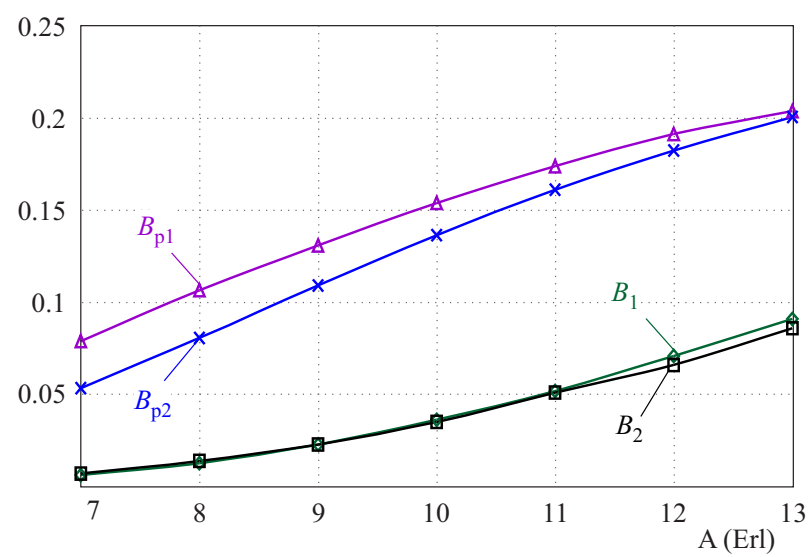

Fig. 7. Loss probabilities: caused by unsuccessful pairing according to the first method ( $\left.B_{p 1}\right)$, according to the second method ( $\left.B_{p 2}\right)$, caused by the lack of idle OSCs in first $\left(B_{1}\right)$ and second $\left(B_{2}\right)$ method when it is $\gamma=3, \Delta l=4 \mathrm{~dB}$

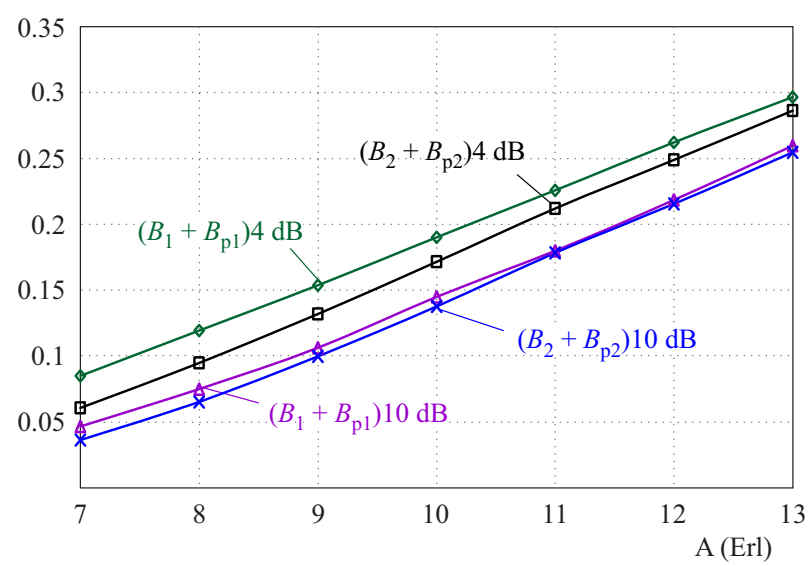

Fig. 9. Total loss probabilities: according to the first method ( $B_{1}+$ $\left.B_{p 1}\right)$, according to the second method $\left(B_{2}+B_{p 2}\right)$, when it is $\Delta_{l}=4$ $\mathrm{dB}$ and $\Delta_{l}=10 \mathrm{~dB}, \gamma=3$

based on generation of $10^{6}$ random numbers related to traffic process simulation (generating new calls and their releasing). In this way it is achieved that each simulation trial consists of more than 350000 realized connections. After that, each result in simulation ( $i e$ each emphasized point in the figures 7-10) is obtained as a mean value of such three described simulation trials. Besides generating random numbers related to the traffic process, for each initiated connection one more generated random number is intended to determine mutual distance between base station and mobile user. In this simulation program this random number is used to define in which of 15 annuli is mobile user placed. After that OSC is seized to simulate connection realization. The difference when simulating two considered models is only in sequence of activities, ie whether we are first looking for an idle timeslot to seize one of two OSCs, or we are first trying to realize pairing, ie seizing OSC in a timeslot where one OSC is already busy. Simulation program keeps record about the ordinal number of an annulus, where the considered mo-

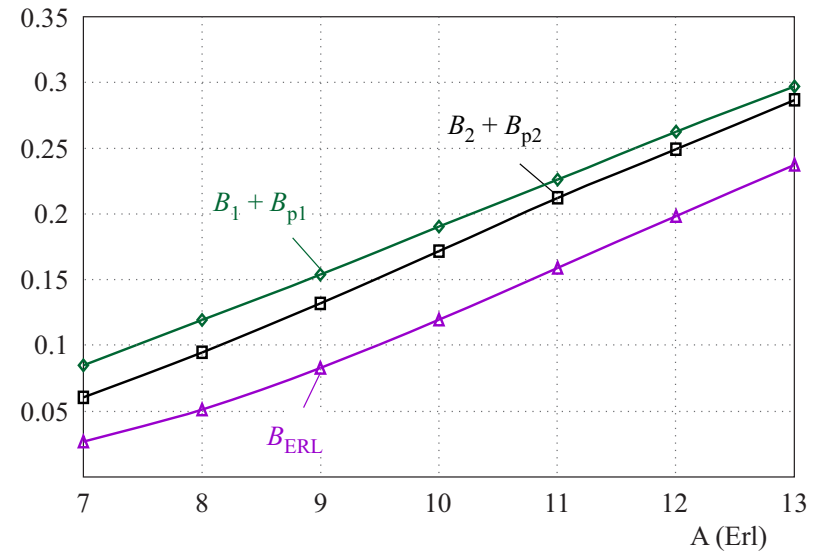

Fig. 8. Total loss probabilities: according to the first method $\left(B_{1}+B_{p 1}\right)$, according to the second method $\left(B_{2}+B_{p 2}\right)$, and loss probability in Erlang model $B_{E}$ under the same conditions $\gamma=3, \Delta l=4 \mathrm{~dB}$

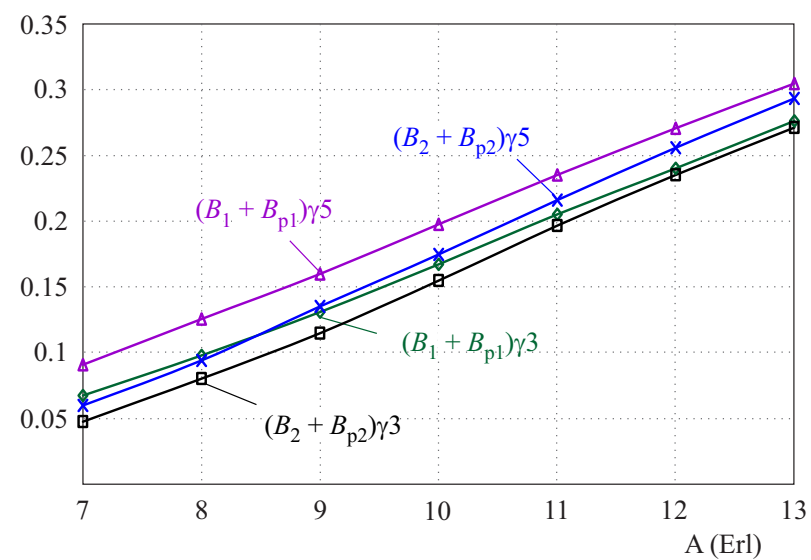

Fig. 10. Total loss probabilities: according to the first method $\left(B_{1}+B_{p 1}\right)$, according to the second method $\left(B_{2}+B_{p 2}\right)$, and loss probability in Erlang model $B_{\mathrm{ERL}}$ under the same conditions $\gamma=3$ and $\gamma=5, \Delta_{i}=6 \mathrm{~dB}$

bile user is placed. When a request for a new connection appears, it is checked what the difference between annuli ordinal numbers is when considering previously realized connection and newly generated call. If this difference is not greater than the pre-defined limit, pairing may be realized in a considered timeslot.

\section{Conclusion}

The first method of OSC assignment gives priority to the connection quality, because OSC selection in idle timeslot has priority over pairing, but the traffic efficiency is smaller. On the contrary, the second method, which first tries pairing and then seizing OSC in idle timeslot, offers lower connection quality, but better resource utilization. The difference in connection quality is described in [2] and in this paper it is proved that difference in traffic efficiency depends on offered traffic, difference in allowed emission power for paired connections and signal attenuation coefficient. 
The loss difference due to unsuccessful pairing in favour of the second model is moderate and it is decreased when traffic is increased. Loss existence due to unsuccessful pairing causes decrease of loss due to the lack of idle OSCs.

Efficiency advantage of the second method also decreases when the allowed emission power difference increases. In this case loss due to unsuccessful pairing decreases, but loss due to the lack of idle OSCs increases.

The greater signal attenuation coefficient causes traffic loss increase due to unsuccessful pairing, while loss ratio of the first and the second method remains the same.

It can be said that the first method is more suitable for small traffic values and the second one for greater traffic values.

\section{REFERENCES}

[1] K. Jung and R. M. Merasereau, Media and Radio Signal Processing for Mobile Communications, Cambridge University Press, March 2018.

[2] M. Kottkamp, "VAMOS Technology Introduction", Application Note, Rohde \& Schwarz, Aug. 2011,https://cdn.rohde-schwarz. com/pws/dl_downloads/dl_application/application_notes/1ma1 81/ 1MA181_2E_VAMOS_technology.pdf.

[3] Recommendation ETSI TS 145004 V13. 2. 0: Digital Cellular telecommunications system (Phase 2+) (GSM); Modulation (3GPP TS 45. 004 version 13. 2. 0 Release 13), August 2016.

[4] M. Säily, G. Sébire and E. Riddington, GSM/EDGE Evolution and Performance, Wiley \& Sons, 2011.

[5] J. Ebersprächer, H.-J. Vögel and Ch. Bettstetter, GSM Switching, Services and Protocols, John Wiley \& Sons, 1999.

[6] ITU-T Recommendation Q. 543: Digital Exchange Performance Design Objectives, March 1993.

[7] M. A. Ruder, User Pairing for Mobile Communication Systems with $O S C$ and SC-FDMA Transmission, Dissertation, Friedrich-Alexander-Universität, 2014.

[8] G. Heine, GSM Networks: Protocols, Terminology and Implementation, Artech House, (1999).

[9] 3rd Generation Partnership Project; Technical Specification Group GSM/EDGE Radio Access Network; Radio subsystem link control (Release 10), 3GPP TS45. 008 v10. 0.0, 01-Mar-11.

[10] V. B. Iversen, "Teletraffic Engineering and Network Planning", Technical University of Denmark, 2015.

[11] M. Šuh, T. Mileusnić, A. Lebl, D. Mitić and Ž. Markov, "Use of Computer Simulation Estimation of GSM Base Station Output Power", Acta Polytechnica Hungarica, vol. 11, no. 6, August 2014, pp. 129-142.

[12] D. Mitić, A. Lebl, M. Mileusnić, B. Trenkić and Ž. Markov, "Traffic Simulation of GSM Cells with Half-Rate Connection Realization Possibility", Journal of Electrical Engineering, vol. 67, no.2, April 2016, pp. 95-102.

[13] M. Mileusnić, P. Jovanović, M. Popović, A. Lebl, D. Miti’c and Ž. Markov, "Influence of Intra-cell Traffic on the Output Power of Base Station GSM", Radioengineering, vol. 23, no.2, June 2014, pp. 601-608.

14] A. Lebl, D. Mitić, M. Popović, Ž. Markov, M. Mileusnić and V. Matić, "Influence of mobile users' density distribution on the CDMA base station power", Journal of Electrical Engineering, vol. 67, no.6, December 2016, pp. 390-398.

Received 19 May 2017

Aleksandar Lebl was born in Zemun, Serbia, in 1957. He received his BSc and MSc from the Faculty of Electrical Engineering in Belgrade, Republic of Serbia, in 1981 and 1986, respectively, and his PhD from the Faculty of Technical Science in Novi Sad, in 2009. He is employed from 1981 in the Switching Department of Institute for Electronics and Telecommunications IRITEL in Belgrade. During years he worked on the project of Digital Switching System for Serbian Telecommunication Industry.

Dragan Mitić was born in Belgrade, Serbia, in 1953. He received his BSc and MSc from the Faculty of Electrical Engineering in Belgrade, Republic of Serbia, in 1977 and 1984, respectively, and his $\mathrm{PhD}$ from the Faculty of Technical Science in Novi Sad, in 2002. Dr Mitić is a senior research fellow in IRITEL, Institute for Electronics and Telecommunications, Belgrade, Serbia. From 1977. until 1989. he was employed at the Land Forces Military Technical Institute in Belgrade, and since 1989. in IRITEL. Dr Mitić is author or co-author of more than 150 international and national scientific and professional papers. He works on several research projects for equipment of specific applications.

Žarko Markov was born in Žitište, Serbia, in 1946. He received his $\mathrm{Bc}, \mathrm{MSc}$ and $\mathrm{PhD}$ from the Faculty of Electrical Engineering in Belgrade, Republic of Serbia, in 1969, 1975 and 1976, respectively. Dr Markov is a scientific counsellor in IRITEL, Institute for Electronics and Telecommunications, Belgrade, Serbia. Area of work: Switching technics, Teletraffic theory, Network signalling. Author or co-author of more than hundred papers and six books. At the University of Belgrade, School of Electrical Engineering, Dr. Markov was a professor at the course of Switching technics and Network signalling.

Mihailo Stanić was born in Bitola, Former Yugoslav Republic of Macedonia, in 1970. He received his Bc, MSc and $\mathrm{PhD}$ from the Faculty of Electrical Engineering in Belgrade, Republic of Serbia, in 1995, 2002 and 2013, respectively. From 1996 Dr Stanić is employed in the Optical Transmission Systems Department of Institute for Electronics and Telecommunications IRITEL in Belgrade. Author or coauthor of more than 30 scientific and professional papers and one scientific monograph. He worked on many research and development projects in area of telecommunication networks management, communication protocols and software systems of various applications. Research interests are in network management and network modelling. 